\title{
Prevalence of a Shared Isthmus in Postinfarction Patients with Pleiomorphic, Hemodynamically Tolerated Ventricular Tachycardias
}

\author{
FRANK BOGUN, M.D., YI-GANG LI, M.D., $\dagger$ GERIAN GROENEFELD, M.D., $\dagger$ \\ STEFAN H. HOHNLOSER, M.D., $\dagger$ CLAUDIO SCHUGER, M.D., HAKAN ORAL, M.D.,* \\ FRANK PELOSI, M.D., * BRADLEY KNIGHT, M.D.,* \\ S. ADAM STRICKBERGER, M.D., ${ }^{*}$ and FRED MORADY, M.D.*
}

From the Division of Cardiology, Henry Ford Hospital, Detroit, Michigan; the *University of Michigan Medical Center, Ann Arbor, Michigan; and the †Division of Cardiology, J.W. Goethe University, Frankfurt, Germany

\begin{abstract}
Shared Isthmus. Introduction: Multiple forms of ventricular tachycardia (VT) after myocardial infarction may result from multiple reentrant circuits that share an isthmus or from separate reentrant circuits. The prevalence of a shared isthmus in patients with multiple hemodynamically tolerated VTs has not been determined.

Methods and Results: Criteria for a shared isthmus consisted of (1) concealed entrainment of $>1$ VT at a single pacing site; (2) concealed entrainment during VT and a perfect pace map of another VT at the same pacing site; or (3) concealed entrainment of VT of a given morphology that had at least two cycle lengths that varied by at least 100 msec. In a series of 19 patients $(16$ men and 3 women; age $65 \pm 14$ years, ejection fraction $0.25 \pm 0.09$ ) with 54 VTs (mean cycle length $494 \pm 98 \mathrm{msec}$ ), there was evidence of a shared isthmus in 23 VTs $(43 \%)$ at 11 sites in 9 patients. Concealed entrainment of two different VTs was observed at 4 of 11 sites. At 5 of 11 sites there was concealed entrainment of one VT and a perfect pace map of another VT. At the remaining 2 of 11 sites, there was concealed entrainment of a VT that had two different cycle lengths. Nineteen of the 23 VTs were ablated successfully with radiofrequency energy applications at 11 sites.

Conclusion: In postinfarction patients with pleiomorphic, hemodynamically stable VT, a shared isthmus may be present in approximately $40 \%$ of VTs. ( $J$ Cardiovasc Electrophysiol, Vol. 13, pp. 237-241, March 2002)
\end{abstract}

ventricular tachycardia, concealed entrainment, pace mapping

\section{Introduction}

Multiple forms of ventricular tachycardia (VT) in a patient with prior myocardial infarction may result from multiple reentrant circuits that share an isthmus or from different reentrant circuits. The prevalence of a shared isthmus in patients with multiple, hemodynamically tolerated VTs has not been determined. The aim of this study was to determine how often an isthmus was present in a consecutive series of postinfarction patients with pleiomorphic, hemodynamically stable VTs.

\section{Methods}

\section{Patient Characteristics}

The subjects of this study were 19 patients (16 men and 3 women; mean age $65 \pm 14$ years [ \pm SD] ) who had at least two forms of inducible, sustained, hemodynamically tolerated VT. These patients were selected from a larger group of 36 patients who were referred for VT ablation. Mean left ventricular ejection fraction was $0.25 \pm 0.09$. All patients

Address for correspondence: Frank Bogun, M.D., Henry Ford Hospital, Division of Cardiology K-14, 2799 West Grand Boulevard, Detroit, MI 48202-9888. Fax: 734-665-5371; E-mail: bogun@eudoramail.com

Manuscript received 31 October 2001; Accepted for publication 5 February 2002. had a history of at least one myocardial infarction (anterior in 7 , inferior in 2, and both anterior and inferior in 10). Indications for catheter ablation were incessant VT in 5 patients; frequent internal cardioverter defibrillator discharges in 7; and recurrent, drug-refractory VT in 7.

A total of 54 VTs with a mean cycle length of $494 \pm 98$ msec were targeted for ablation; 23 had a left bundle branch block morphology and 31 had a right bundle branch block morphology. Eighteen of the patients also were subjects of a previous study. ${ }^{1}$

\section{Electrophysiologic Study and Mapping}

After obtaining informed consent, a 6-French electrode catheter was introduced into the right femoral vein and positioned in the right ventricular apex. A 7-French quadripolar catheter was introduced into a femoral artery and advanced into the left ventricle using a retrograde aortic approach. A 5,000-unit bolus of heparin was administered intravenously, followed by 1,000 units/hour. A quadripolar electrode catheter (Webster, Mansfield, or EP Technologies,) with a 4-mm tip electrode and 2-5-2 $\mathrm{mm}$ interelectrode spacing was used for mapping and ablation. Electrodes 1 and 3 were used for bipolar pacing, and electrodes 2 and 4 were used for bipolar recordings filtered at 50 to 500 $\mathrm{Hz}$. Intracardiac electrograms and leads $\mathrm{V}_{1}$, I, II, and III were displayed on an oscilloscope and recorded using a 
$\mathbf{A}$

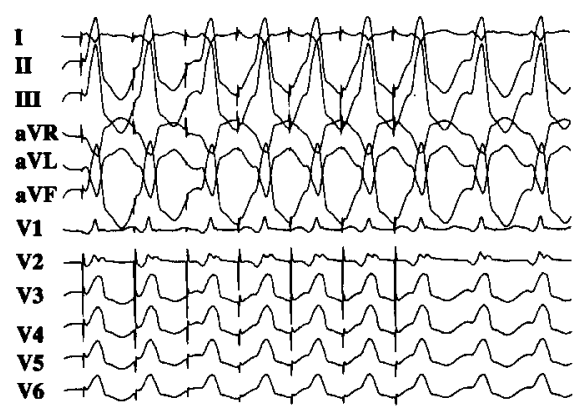

$1000 \mathrm{msec}$

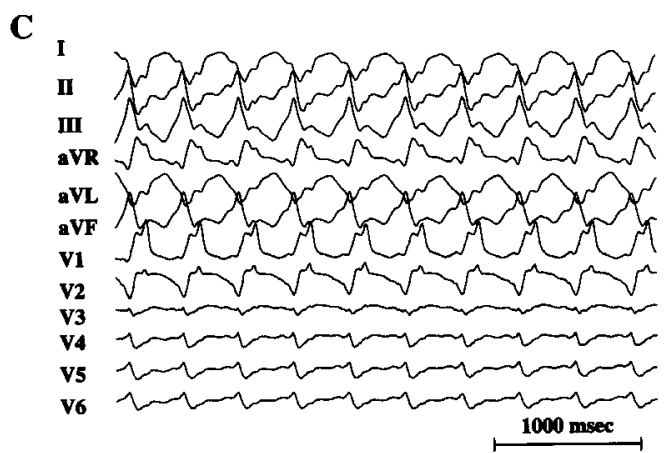

B

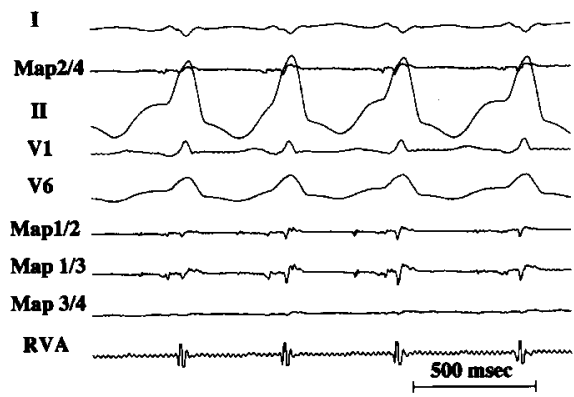

D

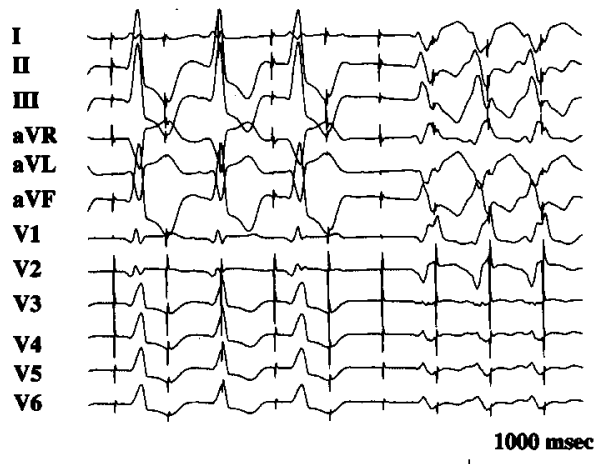

Figure 1. (A) Twelve-lead ECG of ventricular tachycardia (VT) with a cycle length 460 msec. A pacing train at a cycle length of 400 msec was introduced at the basal left ventricular free wall. There is concealed entrainment, with a stimulus-QRS interval of 115 msec. (B) Leads I, II, $V_{1}$, and $V_{\sigma}$, and intracardiac recordings from the mapping catheter (Map2/4, 1/2, 1/3, and 3/4) and right ventricular apex (RVA) during the same VT shown in panel A. The mapping catheter was positioned at the same site where pacing was performed in panel A. The mapping catheter recorded an isolated diastolic potential that preceded the QRS complex by $115 \mathrm{msec}$, similar to the stimulus-QRS interval during concealed entrainment. (C) A second VT that was reproducibly inducible in the same patient. (D) Twelve-lead ECG during pacing in sinus rhythm at the same site as shown in panels A and B. There are two distinct QRS morphologies in response to pacing. The first morphology is that of left bundle branch block and is almost identical to the VT shown in panels $A$ and B. The second morphology is that of right bundle branch block and matches the VT shown in panel C. There initially was 2:1 capture during pacing, then 1:1 capture and a stimulus-QRS interval of $280 \mathrm{msec}$.

Mingograf-7 recorder (Siemens) at a paper speed of 100 $\mathrm{mm} / \mathrm{sec}$. The recordings were stored on optical disk.

If VT was not incessant, VT was induced by right ventricular programmed stimulation with 1 to 4 extrastimuli. ${ }^{2}$ If the VT was hemodynamically stable, left ventricular mapping was performed. At all sites with an abnormal ventricular electrogram (amplitude $\leq 0.5 \mathrm{mV}$ and duration $\geq 60$ $\mathrm{msec}$ ), the mapping catheter was used to introduce pacing trains of 10 to 15 stimuli at cycle lengths 20 to $100 \mathrm{msec}$ shorter than the VT cycle length. The output of the stimulator was increased to $10 \mathrm{~mA}$, and the pulse width was increased to $9 \mathrm{msec}$ as needed to capture the ventricle during VT. Additional maneuvers were performed only at sites where there was concealed entrainment. Pacing at the tachycardia cycle length was performed during sinus rhythm at the same site where concealed entrainment was demonstrated during VT. If an isolated diastolic potential or discrete systolic potential ${ }^{3}$ was present during VT, pacing was performed to determine whether the potential could be dissociated from the VT. Endocardial activation time and stimulus-QRS interval during concealed entrainment were compared to differentiate bystander areas from the isthmus of the reentrant circuit. ${ }^{4}$

\section{Radiofrequency Ablation}

Radiofrequency energy was applied only at sites where there was concealed entrainment. Concealed entrainment was defined as entrainment of VT at multiple pacing cycle lengths, without fusion of the QRS complex. Radiofrequency energy was delivered as a continuous, unmodulated sine wave at a frequency of $500 \mathrm{kHz}$ (EP Technologies). Applications of radiofrequency energy were titrated to maintain a target temperature of $60^{\circ} \mathrm{C}$ at the electrode-tissue interface and were delivered during VT. All applications were continued for at least 20 seconds if adequate heating at the electrode-tissue interface was achieved. If VT stopped within 20 seconds, energy application was continued for 60 seconds. After ablation, programmed ventricular stimulation was repeated. Successful catheter ablation was defined as termination of VT by an application of radiofrequency energy and the inability to reinduce the VT by programmed stimulation.

\section{Data Analysis}

Any one of the following three observations was considered evidence of a shared isthmus: (1) concealed entrainment of one VT and a perfect pace map of a second VT at the same pacing site (Fig. 1); (2) concealed entrainment of two different VTs at a single pacing site (Fig. 2); or (3) concealed entrainment at the same pacing site of VTs that had the same QRS morphology but cycle lengths that differed by at least $100 \mathrm{msec}$ (Fig. 3). 

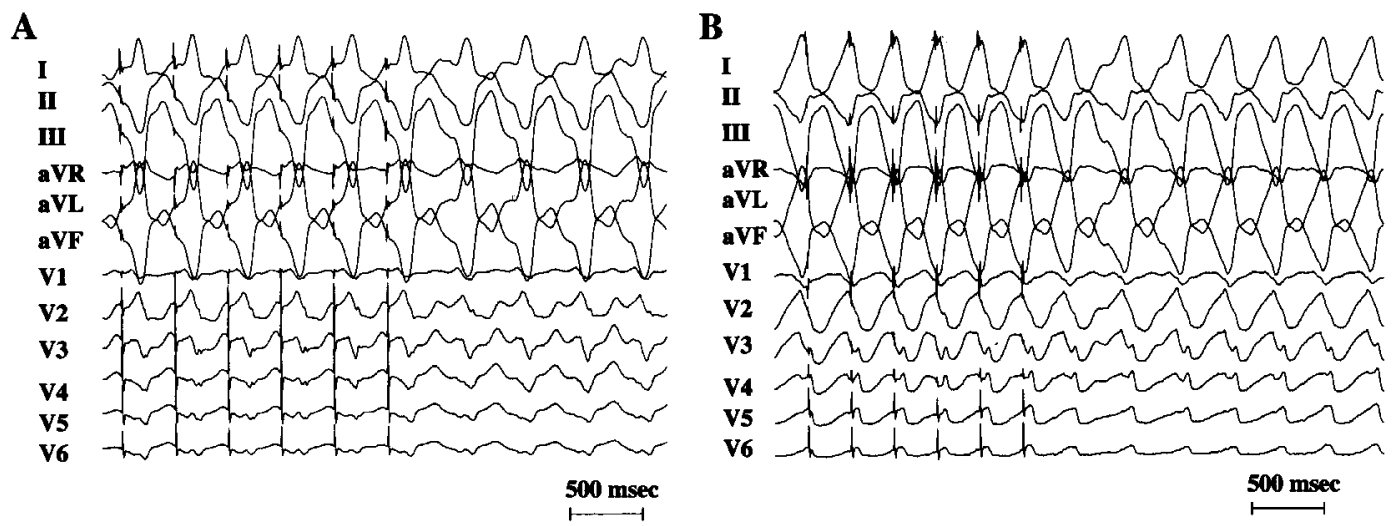

Figure 2. (A) Twelve-lead ECG of ventricular tachycardia (VT) with a cycle length 420 msec. The mapping catheter is located at a left ventricular septal site. Pacing was performed with a cycle length of $370 \mathrm{msec}$. There is concealed entrainment. (B) Another VT with a cycle length of $360 \mathrm{msec}$ at the same septal site. There also is concealed entrainment (pacing cycle length $300 \mathrm{msec}$ ). The QRS morphologies of both the uninterrupted VTs and the paced QRS complexes show minor variations.

\section{Statistical Analysis}

Variables are expressed as mean $\pm 1 \mathrm{SD}$. Variables were compared by Student's $t$-test, Fisher exact test, or Chisquare analysis, as appropriate. $\mathrm{P}<0.05$ was considered statistically significant.

\section{Results}

\section{Shared Isthmus}

Among the 54 VTs, 23 (43\%) met one of the criteria for a shared isthmus at 11 sites in 9 of 19 patients (Table 1). Concealed entrainment of $\geq 2$ VTs occurred at 4 of the 11 sites. Concealed entrainment of 1 or 2 VTs and a perfect pace map of another VT occurred at 5 of the 11 sites. At 2 of the 11 sites, there was concealed entrainment of 2 VTs that had the same morphology but different cycle lengths.

Patients with VTs that had a shared isthmus tended to have a lower ejection fraction than the other patients $(0.21 \pm 0.07$ vs $0.29 \pm 0.09 ; \mathrm{P}=0.06)$ (Table 2). All nine patients with VTs that had a shared isthmus had a history of an anterior infarction. None of the patients with inferior infarction alone had evidence of a shared isthmus.

\section{Mapping Criteria}

Isolated diastolic potentials and discrete systolic potentials were more prevalent at sites with a shared isthmus compared with sites where there was concealed entrainment but no evidence of a shared isthmus (Table 3). Likewise, matching stimulus-QRS and electrogram-QRS intervals were more common at these sites. The endocardial activation time was earlier at sites with features of a shared isthmus compared with other sites. Sites of a shared isthmus also tended to have better pace maps compared with other sites. Other mapping parameters, including the stimulusQRS interval and the ratio of stimulus-QRS to VT cycle length, were similar when sites with and sites without a shared isthmus were compared.

\section{Ablation Results}

Applications of radiofrequency energy at 11 sites where there was a shared isthmus were successful in ablating 19 (83\%) of the 23 VTs (Tables 1 and 3). At 8 of the 11 sites, all VTs with a shared isthmus were ablated. At 2 of 11 sites, only 1 of 2 VTs with a shared isthmus was ablated. At 1 of the 11 sites, none of the 2 VTs with a shared isthmus were ablated.

\section{Discussion}

\section{Main Findings}

The results of this study suggest that a shared isthmus may account for pleiomorphic VTs in almost $50 \%$ of postin-

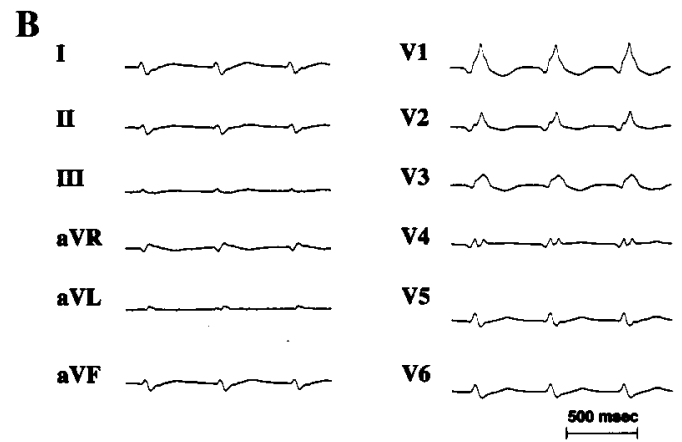

Figure 3. (A) ECG of a ventricular tachycardia (VT) with a right bundle branch morphology and a cycle length of 440 msec. The VT was recorded at a posterolateral left ventricular site. (B) VT that has the same morphology as the VT shown in panel A, but with a cycle length of 540 msec. 
TABLE 1

Sites with a Shared Isthmus

\begin{tabular}{|c|c|c|c|c|c|c|}
\hline Site & VT & CL & Criteria for SI & IDP/SP & S-QRS/EAT & RFA Outcome \\
\hline \multirow[t]{2}{*}{1} & 1: LBSA & 610 & $\mathrm{CE}$ & Yes & $320 / 300$ & + \\
\hline & 2: RBSA & 480 & $\mathrm{CE}$ & No & $540 / 60$ & - \\
\hline \multirow[t]{2}{*}{2} & 1: RBSA & 525 & $\mathrm{CE}$ & Yes & $390 / 120$ & - \\
\hline & 2: RBSA & 490 & $\mathrm{CE}$ & Yes & $220 / 250$ & + \\
\hline \multirow[t]{3}{*}{3} & 1: LBSA & 360 & Pace map & Yes & NA & + \\
\hline & 2: RBSA & 440 & $\mathrm{CE}$ & Yes & $240 / 240$ & + \\
\hline & 3: LBIA & 500 & $\mathrm{CE}$ & Yes & $270 / 270$ & + \\
\hline \multirow[t]{2}{*}{4} & 1: RBSA & 550 & $\mathrm{CE}$ & Yes & $420 / 410$ & + \\
\hline & 2: RBSA & 630 & $\mathrm{CE}$ & Yes & $380 / 400$ & + \\
\hline \multirow[t]{2}{*}{5} & 1: RBIA & 630 & $\mathrm{CE}$ & Yes & $240 / 220$ & + \\
\hline & 2: RBSA & 540 & Pace map & NA & NA & + \\
\hline \multirow[t]{2}{*}{6} & 1: RBIA & 370 & $\mathrm{CE}$ & Yes & $80 / 60$ & + \\
\hline & 2: RBIA & 650 & Pace map & Yes & $-/ 160$ & + \\
\hline \multirow[t]{2}{*}{7} & 1: RBIA & 490 & $\mathrm{CE}$ & Yes & $100 / 110$ & + \\
\hline & 2: RBIA & 370 & Pace map & NA & NA & + \\
\hline \multirow[t]{2}{*}{8} & 1: RBSA & 500 & $\mathrm{CE}$ & No & $380 / 50$ & - \\
\hline & 2: RBIA & 415 & Pace map & NA & NA & - \\
\hline \multirow[t]{2}{*}{9} & 1: LBSA & 420 & $\mathrm{CE}$ & Yes & $50 / 60$ & + \\
\hline & 2: LBSA & 360 & $\mathrm{CE}$ & Yes & $160 / 170$ & + \\
\hline \multirow[t]{2}{*}{10} & 1: RBSA & 440 & $\mathrm{CE}$ & Yes & $350 / 320$ & + \\
\hline & 2: RBSA & 540 & $\mathrm{CE}$ & Yes & $330 / 350$ & + \\
\hline \multirow[t]{2}{*}{11} & 1: RBIA & 490 & $\mathrm{CE}$ & Yes & $130 / 130$ & + \\
\hline & 2: RBIA & 600 & $\mathrm{CE}$ & Yes & $130 / 130$ & + \\
\hline
\end{tabular}

$\overline{\mathrm{CE}}=$ concealed entrainment; $\mathrm{CL}=$ cycle length; IDP = isolated diastolic potential; LBIA = left bundle branch block with inferior axis; LBSA = left bundle branch block with superior axis; NA = not applicable; RBIA = right bundle branch block with inferior axis; RBSA = right bundle branch block with superior axis; RFA = radiofrequency ablation $(+=$ successful, $-=$ unsuccessful $)$; $\mathrm{SI}=$ shared isthmus; $\mathrm{SP}=$ systolic potential; $\mathrm{S}-\mathrm{QRS} / \mathrm{EAT}=$ stimulus-QRS interval/endocardial activation time; VT $=$ ventricular tachycardia.

farction patients who have hemodynamically tolerated VTs. Among patients with a history of an anterior myocardial infarction, approximately $60 \%$ of patients with pleiomorphic VTs have evidence of a shared isthmus. Once concealed entrainment of a VT is demonstrated, evidence of a shared isthmus can be uncovered by pacing at the same site during other VTs or during sinus rhythm. Applications of radiofrequency energy at sites of a shared isthmus usually are effective in ablating the pleiomorphic VTs. Therefore, recognition of a shared isthmus may obviate the need for mapping and ablation of individual VTs.

\section{Anatomic Substrate}

The degree of left ventricular dysfunction was not evaluated in prior reports describing a shared isthmus.,.$^{5,6}$ Intraoperative mapping studies demonstrated complex reentrant circuits, including multiple entry and multiple exit sites, in postinfarction patients with VT. ${ }^{5,6}$ It has been suggested that large areas of the subendocardium participate in an extensive mesh of interwoven muscle bundles to generate multiple, linked reentrant circuits. ${ }^{6}$ This may explain why the patients in this study who had a shared isthmus tended to

\section{TABLE 2}

Characteristics of VTs With and Without a Shared Isthmus

\begin{tabular}{lccc}
\hline Characteristic & VTs With SI & VTs Without SI & P Value \\
\hline No. of sites & $11 / 72$ & $61 / 72$ & \\
No. of patients & $9 / 19(47 \%)$ & $10 / 19(53 \%)$ & \\
No. of VTs & $23 / 54(43 \%)$ & $31 / 54(57 \%)$ & \\
EF & $0.21 \pm 0.07$ & $0.29 \pm 0.09$ & 0.06 \\
VT CL (msec) & $501 \pm 93$ & $489 \pm 102$ & 0.6 \\
Anterior infarction & $9 / 19$ & $6 / 19$ & 0.08 \\
\hline
\end{tabular}

$\overline{\mathrm{EF}}=$ ejection fraction. Other abbreviations as in Table 1. have more extensive left ventricular dysfunction than patients in whom there was no evidence of a shared isthmus. This also corresponds to the higher prevalence of shared isthmus ventricular tachycardias in patients with anterior myocardial infarctions versus inferior myocardial infarctions.

At two sites with shared isthmus VTs, a VT could be induced with both right and left bundle branch block morphology. None of these VTs satisfied the criteria of VTs that can be ablated at the mitral valve isthmus region after inferior wall myocardial infarction. ${ }^{7}$ Therefore, an alternate mechanism is responsible for this observation, and more detailed mapping is required to clarify the underlying mechanism.

\section{Isolated Diastolic Potentials}

Based on the presence of isolated diastolic potentials at a single endocardial site during different VTs, Fitzgerald et

TABLE 3

Mapping Criteria at Sites Showing Features of a Shared Isthmus Compared With Other Sites

\begin{tabular}{lccc}
\hline Criteria & VTs With SI & VTs Without SI & P Value \\
\hline No. of VTs & 23 & 31 & \\
No. of sites & $11 / 72$ & $61 / 72$ & \\
Effective ablations & $19 / 23$ & $29 / 31$ & 0.9 \\
IDPs/SPs per site & $10 / 11$ & $21 / 61$ & $<0.01$ \\
S-QRS per VT & $247 \pm 141$ & $213 \pm 141$ & 0.4 \\
EAT per VT & $201 \pm 116$ & $124 \pm 76$ & $<0.01$ \\
Pace map identical & & & 0.1 \\
$\quad$ per site & $6 / 11$ & $15 / 61$ & 0.6 \\
S-QRS pace map & $119 \pm 56$ & $112 \pm 48$ & \\
\hline
\end{tabular}

Abbreviations as in Tables 1 and 2. 
al. ${ }^{8}$ concluded that a shared isthmus was present. However, pacing was not performed with the ablation catheter to determine whether the diastolic potentials could be dissociated from VT. In the present study, isolated diastolic potentials that were recorded at sites of concealed entrainment could not be dissociated from VT by pacing maneuvers, indicating that the diastolic potentials were recorded within a critical isthmus.

Isolated diastolic potentials were found to be more prevalent at recording sites that were within a shared isthmus than at recording sites within an isthmus that was not shared by different VTs. A previous study demonstrated that isolated diastolic potentials that cannot be dissociated from the VT are markers of an isthmus bounded by anatomically determined regions of block that are present during sinus rhythm as well as during VT. ${ }^{9}$ This provides the rationale for using the combination of concealed entrainment during VT and a perfect pace map of another VT during sinus rhythm as a criterion for a shared isthmus.

\section{Study Limitations}

One limitation of this study is that the results may be applicable only to the subset of patients with postinfarction VT that is hemodynamically stable. Because all of the patients in this study had hemodynamically stable VT and sites of demonstrable concealed entrainment, the results may not apply to other subsets of patients with VT. Not all of the VTs with a shared reentrant circuit were ablated with single radiofrequency energy ablations. It is possible that in these patients, the shared component of the reentrant circuit was critical for one VT but was only a bystander for the other VT. Comparison of the stimulus-QRS intervals with the electrogram-QRS interval at these sites supports this hypothesis (Table 1). In addition, single radiofrequency lesions may not be large enough to interrupt the reentrant circuit even if the lesions are within a critical portion of the reentrant circuit. Because pacing during sinus rhythm at some sites might have resulted in capture at the entry site, it is possible that the criteria for shared isthmus VTs might have resulted in some underestimation of the prevalence of shared isthmus VTs. However, a similar prevalence with respect to shared diastolic pathways and shared exit sites has been reported with a noncontact mapping system. ${ }^{10}$

\section{Conclusion}

A shared isthmus may account for nearly half of the pleiomorphic, hemodynamically stable VTs that are inducible in postinfarction patients and can be detected by the response to pacing during VT and during sinus rhythm at the same site, during different VTs. The presence of a shared isthmus may facilitate ablation of multiple morphologies of hemodynamically tolerated VTs.

\section{References}

1. Bogun F, Knight B, Goyal R, Strickberger SA, Hohnloser SH, Morady $F$ : Clinical value of the postpacing interval for mapping of ventricular tachycardia in patients with prior myocardial infarction. J Cardiovasc Electrophysiol 1999;10:43-51.

2. Hummel D, Strickberger S, Daoud E, Niebauer M, Bakr O, Man K, Williamson B, Morady F: Results and efficency of programmed ventricular stimulation with four extrastimuli compared with one, two, and three extrastimuli. Circulation 1994;90:2827-2823.

3. Bogun F, Knight B, Goyal R, Michaud GF, Strickberger SA, Hohnloser SH, Morady F: Discrete systolic potentials during ventricular tachycardia in patients with prior myocardial infarction. J Cardiovasc Electrophysiol 1999;10:364-369.

4. Bogun F, Bahu M, Knight B, Weiss R, Paladino W, Harvey M, Goyal R, Daoud E, Man K, Strickberger S, Morady F: Comparison of effective and ineffective target sites that demonstrate concealed entrainment in patients with coronary artery disease undergoing radiofrequency ablation of ventricular tachycardia. Circulation 1997;95: 183-190.

5. Downar E, Saito J, Doig JC, Chen TC, Sevaptsidis E, Masse S, Kimber S, Mickleborough L, Harris L: Endocardial mapping of ventricular tachycardia in the intact human ventricle. III. Evidence of multiuse reentry with spontaneous and induced block in portions of reentrant path complex. J Am Coll Cardiol 1995;25:1591-1600.

6. Downar E, Kimber S, Harris L, Mickleborough L, Sevaptsidis E, Masse E, Chen T, Gena A: Endocardial mapping of ventricular tachycardia in the intact human heart, II. Evidence for multiuse reentry in a function sheet of surviving myocardium. J Am Coll Cardiol 1992;20: 869-878.

7. Wilber DJ, Kopp DE, Glascock DN, Kinder CA, Kall JG: Catheter ablation of the mitral isthmus for ventricular tachycardia associated with inferior infarction. Circulation 1995;92:3481-3489.

8. Fitzgerald D, Friday K, Yeung-Lai-Wah J, Bowman A, Lazzara R, Jackman W: Myocardial regions of slow conduction participating in the reentrant circuit of multiple ventricular tachycardias: Report on ten patients. J Cardiovasc Electrophysiol 1991;2:193-206.

9. Bogun F, Bahu M, Knight BP, Weiss R, Goyal R, Daoud E, Man KC, Strickberger SA, Morady F: Response to pacing at sites of isolated diastolic potentials during ventricular tachycardia in patients with previous myocardial infarction. J Am Coll Cardiol 1997;30:505-513.

10. Chow A, Schilling R, Peter N, Davies D: Ablation of ventricular tachycardia in patients with implantable defibrillators: Advantages of using a non-contact system. Circulation 2000;102:A-3008. 